\title{
Psychometric Properties of the Chinese Version of the Bipolar Depression Rating Scale for Bipolar Disorder
}

This article was published in the following Dove Press journal:

Neuropsychiatric Disease and Treatment

\author{
Jing-Xu Chen ${ }^{1, *}$ \\ Lu Yin ${ }^{l} * *$ \\ Hai-Ting $X u^{\prime}$ \\ Suo-Yuan Zhang' \\ Wen-Qian Huang \\ Hong-Juan Li' \\ Bin-Bin $\mathrm{Li}^{\prime}$ \\ Ke-Bing Yang' \\ Qian $\mathrm{Li}^{2}$ \\ Michael Berk ${ }^{3}$ \\ Yun-Ai Su iD ${ }^{2}$ \\ 'Beijing HuiLongGuan Hospital, Peking \\ University HuiLongGuan Clinical Medical \\ School, Beijing, 100096, People's Republic \\ of China; ${ }^{2}$ Peking University Sixth \\ Hospital, Peking University Institute of \\ Mental Health, NHC Key Laboratory of \\ Mental Health (Peking University), \\ National Clinical Research Center for \\ Mental Disorders (Peking University \\ Sixth Hospital), Beijing, 100083, People's \\ Republic of China; ${ }^{3}$ Deakin University, \\ IMPACT Strategic Research Centre, \\ School of Medicine, Barwon Health, \\ Geelong, Vic., Australia \\ *These authors contributed equally to \\ this work
}

Correspondence: Yun-Ai Su

Email suyunai@I63.com
Introduction: Unlike unipolar depression, depressive episode of bipolar disorder is often associated with clinical characteristics, such as atypical and mixed symptoms. However, there are currently no valid and reliable specific tools available to assess the specific psychiatric symptomatology of depressive episode of bipolar disorder in China. Therefore, we aimed to evaluate the psychometric properties of the Chinese version of the Bipolar Depression Rating Scale (BDRS) in Chinese patients with bipolar disorder.

Methods: The sample of this study included 111 patients with bipolar disorder (30 male, 81 female). All participants were interviewed with the Chinese version of the BDRS (BDRS-C), the 17-item Hamilton Depression Rating Scale (HAMD-17), the Montgomery-Asberg Depression Rating Scale (MADRS) and the Young Mania Rating Scale (YMRS) A psychometric analysis of the BDRS was conducted.

Results: The Cronbach's alpha coefficient of the BDRS-C reached a value of 0.869 . The BDRS-C score and scores for the HAMD-17 $(\mathrm{r}=0.819, p<0.01)$, the MADRS $(\mathrm{r}=0.882$, $p<0.01)$ and the YMRS $(\mathrm{r}=0.355, p<0.01)$ exhibited significant positive correlations. Close correlations were observed between the mixed subscale score of the BDRS-C and the YMRS score $(\mathrm{r}=0.784, p<0.01)$. Exploratory factor analysis resulted in three factors: a primary depressive symptoms cluster, a secondary depressive symptoms cluster, and a mixed symptoms cluster.

Conclusion: The Chinese version of the BDRS has satisfactory psychometric properties. This is a valid and reliable instrument to assess depressive symptomatology in patients with bipolar disorder.

Keywords: bipolar disorder, mania, depression, psychometrics, depressive episode, rating scale, psychiatry

\section{Introduction}

Bipolar disorder is characterized by recurrent episodes of depression that are typically interspersed with manic or hypomanic symptoms. It accounts for a large proportion of the global burden of disease and is thus a major public health concern. ${ }^{1}$ A worldwide meta-analysis showed that the lifetime prevalence of bipolar disorder was over $2 \%$ in the general adult population, ${ }^{2}$ higher than the corresponding figures in China., ${ }^{3,4}$ Depressive symptoms rather than (hypo)manic symptoms represent the predominant abnormal mood state for patients with both bipolar I and II disorders. ${ }^{5-7}$ The clinical significance of depressive episode of bipolar disorder is underscored by a strong association with a markedly increased risk of suicide. ${ }^{8-10}$ It is recommended that 
clinicians routinely assess depressive symptoms in patients suffering from bipolar disorder. ${ }^{10}$

Making an accurate diagnosis of bipolar disorder is difficult in clinical practice because approximately half of the patients present with depression as their first episode, and depressive episode of bipolar disorder and unipolar depression share similar symptoms. ${ }^{11,12}$ Therefore, it is particularly critical to reveal the distinction between bipolar and unipolar depression, which can help to identify unipolar depression. Recently, some studies confirmed that many of the characteristic symptoms were associated with a depressive episode of bipolar disorder, such as depression with a few manic symptoms (depressive mixed state), psychotic depression, atypical depression and mood lability. ${ }^{13-17}$ In the Diagnostic and Statistical Manual of Mental Disorders Fifth Edition (DSM$5),{ }^{18}$ atypical depression is characterized by an increase in appetite, hypersomnia, leaden paralysis, and a long-standing pattern of interpersonal relationship sensitivity. Clinical psychiatric evaluations optimized for unipolar depression, such as the Montgomery and Asberg Depression Scale (MADRS) ${ }^{19}$ and the 17-item Hamilton Depression Scale (HAMD-17), ${ }^{20}$ have been widely used among patients with a depressive episode of bipolar disorder. However, these tools do not include many symptoms specific to depressive episode of bipolar disorder, such as atypical and mixed features. ${ }^{21,22}$ Thus, utilizing these scales for patients with a depressive episode of bipolar disorder could be misleading in both clinical research studies and clinical practice. Improved understanding of the psychopathological characteristics of bipolar depression and the development of good assessment tools are critical.

A new assessment tool scale called the Bipolar Depression Rating Scale (BDRS), which is the first semi-structured, clinician-rated scale tailored to the symptom profile of depressive episode of bipolar disorder, was established and validated in 2007 by Berk et al. ${ }^{21}$ The BDRS comprises 20 items that are individually evaluated from 0 to 3 and can be divided into two subscales: the depressive subscale and the mixed subscale. In this scale, atypical symptoms such as hypersomnia and hyperphagia, as well as mixed symptoms (eg, increased speech, increased motor drive and agitation), are included. ${ }^{21}$ It is a useful tool not only for assessing depressive symptomatology but also for evaluating the effectiveness of the intervention in the management of depressive episode of bipolar disorder. ${ }^{23-27}$

The BDRS was originally written in English and has so far been successfully translated into approximately 5 languages, including Iranian, ${ }^{28}$ Turkish, ${ }^{29}$ Spanish, $^{30}$ Korean $^{31,32}$ and Italian. ${ }^{33}$ All versions of the BDRS were demonstrated to be valid and reliable tools. As such, the purposes of our study were to translate the English version of the BDRS into Chinese with appropriate cultural adaptation and investigate the reliability and validity of the Chinese version of the BDRS (BDRS-C) for the evaluation of patients with bipolar disorder in China.

\section{Methods \\ Participants}

This study was conducted between February 1, 2019, and December 31, 2019, in Beijing Hui-Long-Guan Hospital, Beijing, China. Participants were recruited from patients suffering from bipolar disorder through convenience sampling. For reliability and validity testing and factor analysis, the sample size should be $5-10$ times the number of items in the scale and should be $>100$ participants. In addition, according to the sample size in studies about other language versions of the scale, ${ }^{21,28-33}$ we set the sample size to 120 . Inclusion criteria included subjects with the following characteristics: age between 18 and 65 years old and meeting the DSM-5 for bipolar disorder by the consensus of two independent senior psychiatrists with the title of an associate chief physician or above. All participants were Chinese. Patients were excluded if they had severe cognitive impairment.

The study protocol was approved by the Human Ethics Committee of Beijing Hui-Long-Guan Hospital, and all subjects provided written informed consent according to the Declaration of Helsinki.

\section{Procedure}

The original BDRS is an interview-based tool. This scale contains 20 items, with each item rated on a 4-point Likert scale $(0=$ nil, $1=$ mild, $2=$ moderate, and $3=$ severe $)$ based on a manual that includes the description of the characteristics of every item. ${ }^{21}$ The total score ranges from 0 to 60 , with higher scores indicating more severe depression. This scale contains three factors, namely, depression (somatic), depression (psychological) and mixed symptom clusters. The BDRS demonstrated excellent internal consistency (Cronbach's $\alpha=0.917){ }^{21}$

Four steps were undertaken to achieve a high-quality Chinese translation and comparability with the original scale. First, two clinical psychiatrists (Yin L and Yang KB) fluent in English and Chinese independently translated the original scale into Chinese and developed two translation versions. Then, both translations were integrated by the translators into a single version by discussion. Next, the expert panel ( $\mathrm{Su}$ YA, Chen JX) revised and modified inconsistent and inaccurate items to obtain the final Chinese version of the BDRS. The expressions of all items were simple and easy to understand and were adapted culturally at a conceptual level across Chinese cultures. The final version was 
back-translated into English by Li BB, who had not read the original version of the BDRS, followed by a consistency check by the corresponding author of the original publication. ${ }^{21}$ The evaluators determined that there were no phenomena that were difficult for the participants to understand.

All investigators participating in this study were experienced psychiatrists with more than 10 years of clinical experience in the field of affective disorders. Four of them assessed all participants by the BDRS-C, the MADRS, the HAMD-17 and the Young Mania Rating Scale (YMRS). ${ }^{34}$ The four raters were trained in a whole-day session with a discussion of each scale and training on cases until the internal concordance reached a value of at least 0.80 .

The interrater reliability of the BDRS-C was established on the basis of ratings of 35 patients who were randomly selected from all the participants by the four raters. Each rater performed an independent evaluation using the BDRS$\mathrm{C}$ scale manual without communication, and one senior physician performed a clinical psychiatric examination on these patients. Thirty-six patients were randomly selected and assessed twice by the same assessor for the BDRS-C intrarater and inter-rater reliability tests at a 5-day interval.

\section{Data Analysis}

To measure the internal consistency of the scale, we used Cronbach's $\alpha$ coefficient defined as follows: $>0.90$ : excellent, 0.8-0.9: good, 0.7-0.8: acceptable, 0.6-0.7: questionable, $0.5-0.6$ : poor, $<0.5$ : unacceptable. $^{32}$ To measure the interrater and intrarater reliability, we computed intraclass correlation coefficients (ICC) with 95\% confidence intervals. Concurrent validity was measured by Pearson's correlation to examine the relationships between the scores of the BDRS-C and the other scales (HAMD-17, MADRS and YMRS). Furthermore, a factor analysis was performed to identify the underlying dimensions and structure of the BDRS-C. The factors were determined using the method of unweighted least squares with varimax factor rotations and a scree plot. All statistical tests were two-tailed, and statistical significance was achieved when $p<0.05$. Data were analyzed using SPSS Statistical Package (version 20.0) for Windows.

\section{Results}

A total of 120 patients with bipolar disorder participated in the study. However, nine of them had more than $10 \%$ missing data (more than two missing items) in the BDRS-C and were excluded from further analyses. Among all 111 participants, the average age was $32.2 \pm 13.5$ years old, and 81 were female (73.0\%). The sample consisted of 6 patients with hypo/manic episodes $(5.4 \%), 80$ patients with depressive episodes $(73.9 \%)$, 14 patients with mixed episodes $(12.6 \%)$ and 11 euthymic patients $(9.9 \%)$. Of these patients, 88 met the criteria for bipolar

Table I Sociodemographic and Clinical Characteristics of the Sample $(n=I \mid I)$

\begin{tabular}{|c|c|}
\hline Variables & Values \\
\hline Age (years, mean $\pm S D$ ) & $32.2 \pm 13.5$ \\
\hline \multicolumn{2}{|l|}{ Gender (n, \%) } \\
\hline Male & 30 (27.0\%) \\
\hline Female & $8 \mathrm{I}(73.0 \%)$ \\
\hline Employed (n, \%) & $63(59.4 \%)$ \\
\hline \multicolumn{2}{|l|}{ Marital status (n, \%) } \\
\hline Never married & 37 (33.3\%) \\
\hline Married & $65(58.6 \%)$ \\
\hline Divorced & $9(8.1 \%)$ \\
\hline \multicolumn{2}{|l|}{ Educational level (n, \%) } \\
\hline Up to 6 years & $17(15.3 \%)$ \\
\hline $7-12$ years & $35(31.5 \%)$ \\
\hline $13-16$ years & $52(46.8 \%)$ \\
\hline Over 16 years & 7 (6.3\%) \\
\hline Onset age (years, mean $\pm S D$ ) & $23.6 \pm 9.4$ \\
\hline Duration of illness (Months, median, IQR) & $77(132)$ \\
\hline \multicolumn{2}{|l|}{ Diagnosis } \\
\hline Bipolar I disorder & $88(79.3 \%)$ \\
\hline Bipolar II disorder & $23(20.7 \%)$ \\
\hline \multicolumn{2}{|l|}{ Mood state (n, \%) } \\
\hline Hypo/manic episode & $6(5.4 \%)$ \\
\hline Euthymic & II (9.9\%) \\
\hline Depressive episode & $80(73.9 \%)$ \\
\hline Mixed episode & $14(12.6 \%)$ \\
\hline \multicolumn{2}{|l|}{ Medication (n, \%) } \\
\hline$M S+A P$ & $20(18.0 \%)$ \\
\hline$M S+A D$ & $15(13.5 \%)$ \\
\hline$A P+A D$ & 9 (8.1\%) \\
\hline$M S+A P+A D$ & $63(56.8 \%)$ \\
\hline AP monotherapy & $3(2.7 \%)$ \\
\hline MS monotherapy & I (0.9\%) \\
\hline \multicolumn{2}{|l|}{ Scales scores } \\
\hline BDRS score (mean \pm SD) & $24.1 \pm 10.04$ \\
\hline HAMD score (median, IQR) & $16(13)$ \\
\hline MADRS score (median, IQR) & $22(18)$ \\
\hline YMRS score (median, IQR) & $3(8)$ \\
\hline
\end{tabular}

Abbreviations: SD, standard deviation; IQR, interquartile range; SSRI, selective serotonin reuptake inhibitors; HAMD, Hamilton Depression Scale; MADRS, Montgomery and Asberg Depression Scale; YMRS, Young Mania Rating Scale; MS, mood stabilizer; AP, antipsychotic; AD, antidepressant. 
Table 2 Internal Consistency Features of the Chinese Version of the BDRS $(n=111)$

\begin{tabular}{|c|c|c|c|c|}
\hline & $\begin{array}{l}\text { Mean Value if Item } \\
\text { Deleted }\end{array}$ & $\begin{array}{l}\text { SD Value if Item } \\
\text { Deleted }\end{array}$ & $\begin{array}{l}\text { Item-Total } \\
\text { Correlation }\end{array}$ & $\begin{array}{l}\text { Cronbach's } \alpha \text { if Item } \\
\text { Deleted }\end{array}$ \\
\hline I.Depression & 22.09 & 89.010 & 0.653 & 0.857 \\
\hline 2.Sleep disturbance & 22.38 & 88.819 & 0.502 & 0.862 \\
\hline 3.Appetite disturbance & 22.72 & 88.421 & 0.494 & 0.862 \\
\hline 4.Social impairment & 22.36 & 87.687 & 0.569 & 0.859 \\
\hline $\begin{array}{l}\text { 5.Activity/energy } \\
\text { reduction }\end{array}$ & 22.27 & 88.199 & 0.613 & 0.858 \\
\hline 6.Reduced motivation & 22.32 & 87.676 & 0.610 & 0.858 \\
\hline $\begin{array}{l}\text { 7.Reduced } \\
\text { concentration }\end{array}$ & 22.27 & 89.708 & 0.508 & 0.862 \\
\hline 8.Anxiety & 22.45 & 88.159 & 0.555 & 0.860 \\
\hline 9.Anhedonia & 22.39 & 87.749 & 0.571 & 0.859 \\
\hline 10.Flattened affect & 22.95 & 90.415 & 0.466 & 0.863 \\
\hline II.Worthlessness & 22.44 & 85.940 & 0.586 & 0.858 \\
\hline 12.Helplessness & 22.56 & 86.849 & 0.623 & 0.857 \\
\hline I3.Suicidal ideation & 23.06 & 88.732 & 0.520 & 0.861 \\
\hline 14.Guilt & 22.45 & 90.159 & 0.465 & 0.863 \\
\hline I5.Psychotic symptoms & 22.94 & 91.223 & 0.422 & 0.865 \\
\hline 16.Irritability & 22.96 & 93.799 & 0.373 & 0.866 \\
\hline 17.Lability & 22.99 & 92.209 & 0.376 & 0.866 \\
\hline $\begin{array}{l}\text { I8.Increased motor } \\
\text { drive }\end{array}$ & 23.55 & 100.468 & -0.094 & 0.878 \\
\hline 19.Increased speech & 23.50 & 99.161 & 0.000 & 0.876 \\
\hline \multirow[t]{2}{*}{ 20.Agitation } & 23.64 & 96.942 & 0.221 & 0.870 \\
\hline & & & & Cronbach's $\alpha$ \\
\hline BDRS-C & & & & 0.869 \\
\hline BDRS-C-D & & & & 0.891 \\
\hline BDRS-C-M & & & & 0.730 \\
\hline
\end{tabular}

Abbreviations: BDRS-C, Bipolar Depression Rating Scale; BDRS-D, the depressive subscale of BDRS-C; BDRS-M, the mixed subscale of BDRS-C.

I disorder (79.3\%) and 23 met the criteria for bipolar II disorder (20.7\%). Relevant sociodemographic and clinical data for the subjects are summarized in Table 1.

Table 2 shows the internal consistency of the BDRS-C. Internal consistency was good, with an overall Cronbach's $\alpha$ coefficient of 0.869 . Good internal consistency was also found in the depressive subscale of the BDRS-C (items 1-15), with Cronbach's $\alpha$ coefficient up to 0.891. In comparison, the mixed subscale (items 16-20) had a lower Cronbach's $\alpha$ coefficient of 0.730 but was acceptable.

As shown in Table 3, Pearson's correlation coefficients between the BDRS-C score and the HAMD-17, MADRS and YMRS scores were identified. The BDRS-C scores were highly associated with the HAMD-17 scores $(\mathrm{r}=$ $0.866, p<0.01)$ as well as the MADRS scores $(r=$ 
Table 3 Correlations Between the Total Scores of the BDRS, HDRS, MADRS, YMRS and Factors $(n=I I I)$

\begin{tabular}{|l|l|l|l|l|l|l|l|l|l|}
\hline & YMRS & HAMD & MADRS & BDRS & BDRS-D & BDRS-M & FactorI & Factor2 & Factor3 \\
\hline YMRS & I & & & & & & & \\
\hline HAMD & $0.254^{* *}$ & 1 & & & & & & \\
\hline MADRS & 0.122 & $0.882^{* *}$ & 1 & & & & & \\
\hline BDRS & $0.355^{* *}$ & $0.819^{* *}$ & $0.866^{* *}$ & 1 & & & & \\
\hline BDRS-C-D & 0.168 & $0.834^{* *}$ & $0.906 * *$ & $0.967^{* *}$ & 1 & & & \\
\hline BDRS-C-M & $0.784^{* *}$ & 0.145 & 0.068 & $0.368^{* *}$ & 0.123 & 1 & & \\
\hline FactorI & 0.161 & $0.803^{* *}$ & $0.875^{* *}$ & $0.947^{* *}$ & $0.974 * *$ & 0.135 & 1 & \\
\hline Factor2 & $0.199 *$ & $0.725^{* *}$ & $0.748^{* *}$ & $0.810^{* *}$ & $0.816^{* *}$ & 0.175 & $0.722^{* *}$ & 1 \\
\hline Factor3 & $0.784^{* *}$ & 0.145 & 0.068 & $0.368^{* *}$ & 0.123 & $1.000^{* *}$ & 0.080 & 0.175 \\
\hline
\end{tabular}

Notes: Factor I: primary depressive symptoms cluster. Factor2: secondary depressive symptoms cluster. Factor3: mixed symptoms cluster. $*$ Correlation is significant at the 0.05 level. **Correlation is significant at the 0.01 level.

Abbreviations: YMRS, Young Mania Rating Scale; HAMD, Hamilton Depression Scale; MADRS, Montgomery Asberg Depression Rating Scale; BDRS, Bipolar Depression Rating Scale; BDRS-C-D, the depressive subscale of the BDRS-C; BDRS-C-M, the mixed subscale of Bipolar Depression Rating Scale.

$0.819, p<0.01)$ but weakly correlated with the scores of the YMRS $(\mathrm{r}=0.355, p<0.01)$. Further analysis showed that the associations between the scores for the depressive subscale of the BDRS-C and the HAMD-17 and MADRS scores were strongly correlated $(\mathrm{r}=0.834, p<0.01 ; \mathrm{r}=$ $0.906, p<0.01$; respectively). Pearson correlation coefficients demonstrated high correlations between the scores for the mixed subscale of the BDRS-C and the YMRS scores $(\mathrm{r}=0.784, p<0.01)$.

The results showed that the Kaiser-Meyer-Olkin (KMO) value, which was calculated as an indicator of data coherence, was 0.824 , and Bartlett's test of sphericity reached a level of significance $\left(\chi^{2}=876.202, p<0.01\right)$, indicating that it was suitable for exploratory factor analysis. An unweighted least squares factor analysis was performed, followed by oblique (Promax) rotations of 2-5 factors. Prior to rotation, the five eigenvalues above 1 were $6.332,2.642,1.393,1.162$ and 1.062 , with corresponding percentages of variance accounted for of $31.660 \%, 13.210 \%, 6.965 \%, 5.812 \%$ and 5.309\%. Based on examination of a scree plot and other international versions of BDRS, ${ }^{21,29-31,33}$ the three-factor solution with an oblique rotation was judged to provide a useful account of the data which explained $51.835 \%$ of the total variance. Table 4 shows the component loading matrix (only loads $>0.30$ ), eigenvalues and percent of variance accounted for each factor. The first factor was named the primary depressive symptoms cluster (Factor 1 ) because it concerned aspects such as depression, activity/energy reduction, reduced motivation, anhedonia, and suicidal ideation. The second factor was labeled the secondary depressive symptoms cluster (Factor 2) because it concerned aspects such as social impairment, anxiety, guilt, and psychotic symptoms. The last factor was labeled the mixed symptoms cluster because it concerned manic/ hypomanic aspects, for example, lability, increased motor drive, increased speech or agitation. The primary and secondary depressive symptom clusters also formed a composite depressive symptom cluster.

The inter-rater reliability for the four physicians across a subgroup of 35 (31.5\%) of 111 participants was assessed using ICC. As shown in Table 5, the inter-rater reliability was high, yielding an ICC of 0.946 (95\% confidence interval [CI] 0.912-0.970); this was also the case for the depressive factor (ICC: 0.961; 95\% CI: 0.936-0.978) and the mixed factor (ICC: $0.799 ; 95 \%$ CI: 0.694-0.881). The results also demonstrated that each item had moderate to high inter-rater reliability, except for item 17 (lability).

Test-retest reliability was performed in the subgroup of 36 participants at five-day intervals (Table 6). The ICC was 0.930 for the total score (95\% CI: $0.880-0.967), 0.955$ for the depressive factor (95\% CI: 0.921-0.979) and 0.791 for the mixed factor (95\% CI: 0.619-0.915). Intra-rater reliability for this assessment was moderate to high for individual items $(\mathrm{ICC}=0.632-0.901)$. 
Table 4 Factor Analysis of the Chinese Version of the BPRS $(n=\mid$ II)

\begin{tabular}{|l|l|l|l|}
\hline & Factor I & Factor 2 & Factor 3 \\
\hline I.Depression & 0.755 & & \\
\hline 2.Sleep disturbance & 0.630 & & \\
\hline 3.Appetite disturbance & 0.582 & & \\
\hline 4.Social impairment & 0.504 & 0.517 & \\
\hline 5.Activity/energy reduction & 0.649 & 0.323 & \\
\hline 6.Reduced motivation & 0.743 & & \\
\hline 7.Reduced concentration & & 0.759 & \\
\hline 8.Anxiety & & 0.657 & \\
\hline 9.Anhedonia & 0.634 & 0.310 & \\
\hline I0.Flattened affect & 0.758 & & \\
\hline I I.Worthlessness & 0.567 & 0.401 & \\
\hline I2.Helplessness & 0.607 & 0.363 & \\
\hline I3.Suicidal ideation & 0.529 & 0.308 & \\
\hline I4.Guilt & & 0.528 & \\
\hline 15.Psychotic symptoms & & 0.708 & \\
\hline I6.Irritability & & 0.314 & 0.599 \\
\hline I7.Lability & & & 0.67 I \\
\hline I8.Increased motor drive & & & \\
\hline I9.Increased speech & & & \\
\hline 20.Agitation & & & \\
\hline Notes Extrion Met & & \\
\hline
\end{tabular}

Notes: Extraction Method: Unweighted Least Squares. Rotation Method: Varimax with Kaiser Normalization. Factor I: primary depressive symptoms cluster. Factor2: secondary depressive symptoms cluster. Factor3: mixed symptoms cluster. Abbreviation: BDRS, Bipolar Depression Rating Scale.

\section{Discussion}

To our knowledge, this is the first study to translate and evaluate the psychometric properties of a scale for evaluating the severity of bipolar depressive symptoms in the Chinese population. The Cronbach's alpha analysis suggests good internal consistency of this instrument $(\alpha=0.869)$. Although the BDRS-C in the patients with bipolar disorder was somewhat lower in comparison with the original BDRS $(\alpha=0.917),{ }^{21}$ the internal consistency is consistent with other validation studies in different sociocultural contexts. For example, Cronbach's alpha was found to be 0.81 in the Iranian
Table 5 Inter-Rater Reliability $(n=35)$

\begin{tabular}{|c|c|c|c|}
\hline & ICC & $(95 \% \mathrm{CI})$ & $\mathbf{p}$ \\
\hline I.Depression & 0.737 & $0.6 I I-0.84 I$ & $<0.001$ \\
\hline 2.Sleep disturbance & 0.766 & $0.650-0.860$ & $<0.001$ \\
\hline 3. Appetite disturbance & 0.817 & $0.719-0.892$ & $<0.001$ \\
\hline 4.Social impairment & 0.814 & $0.716-0.891$ & $<0.001$ \\
\hline 5.Activity/energy reduction & 0.774 & $0.660-0.865$ & $<0.001$ \\
\hline 6.Reduced motivation & 0.827 & $0.733-0.898$ & $<0.001$ \\
\hline 7.Reduced concentration & 0.904 & $0.846-0.945$ & $<0.001$ \\
\hline 8.Anxiety & 0.917 & $0.866-0.953$ & $<0.001$ \\
\hline 9.Anhedonia & 0.924 & $0.877-0.957$ & $<0.001$ \\
\hline 10.Flattened affect & 0.800 & $0.696-0.882$ & $<0.001$ \\
\hline II.Worthlessness & 0.880 & $0.811-0.931$ & $<0.001$ \\
\hline 12.Helplessness & 0.903 & $0.845-0.945$ & $<0.001$ \\
\hline I3.Suicidal ideation & 0.928 & $0.883-0.959$ & $<0.001$ \\
\hline 14.Guilt & 0.796 & $0.689-0.879$ & $<0.001$ \\
\hline I5.Psychotic symptoms & 0.887 & $0.821-0.936$ & $<0.001$ \\
\hline 16.Irritability & 0.706 & $0.57 I-0.820$ & $<0.001$ \\
\hline I7.Lability & 0.544 & $0.379-0.704$ & $<0.001$ \\
\hline 18.Increased motor drive & 0.705 & $0.569-0.819$ & $<0.001$ \\
\hline 19.Increased speech & 0.716 & $0.583-0.827$ & $<0.001$ \\
\hline 20.Agitation & 0.839 & $0.750-0.906$ & $<0.001$ \\
\hline BDRS total score & 0.946 & $0.912-0.970$ & $<0.001$ \\
\hline BDRS-D & 0.961 & $0.936-0.978$ & $<0.001$ \\
\hline BDRS-M (items I6-20) & 0.799 & $0.694-0.88 I$ & $<0.001$ \\
\hline
\end{tabular}

Abbreviations: BDRS, Bipolar Depression Rating Scale; BDRS-D, the depressive subscale of the BDRS (itemsI-15); BDRS-M, the mixed subscale of Bipolar Depression Rating Scale.

version, ${ }^{28} 0.870$ in the Spanish version, ${ }^{30} 0.866$ in the Korean version, ${ }^{31}$ and 0.82 in the Italian version. ${ }^{33}$ Therefore, the results we obtained showed that the BDRS-C is a valid and reliable instrument for measuring the severity of depression in Chinese patients with bipolar disorder. Moreover, the four raters' agreements concerning each item score ranged from 0.544 to 0.917 , which indicates acceptable reliability of the rating of different items. 
Table 6 Intra-Rater Reliability $(n=36)$

\begin{tabular}{|c|c|c|c|}
\hline & ICC & $(95 \% \mathrm{CI})$ & $\mathbf{p}$ \\
\hline I.Depression & $0.77 \mid$ & $0.650-0.861$ & $<0.001$ \\
\hline 2.Sleep disturbance & 0.849 & $0.745-0.826$ & $<0.001$ \\
\hline 3.Appetite disturbance & 0.713 & $0.514-0.839$ & $<0.001$ \\
\hline 4.Social impairment & 0.858 & $0.756-0.929$ & $<0.001$ \\
\hline 5.Activity/energy reduction & 0.802 & $0.693-0.886$ & $<0.001$ \\
\hline 6. Reduced motivation & 0.901 & $0.803-0.957$ & $<0.001$ \\
\hline 7.Reduced concentration & $0.76 \mathrm{I}$ & $0.564-0.887$ & $<0.001$ \\
\hline 8.Anxiety & 0.829 & $0.717-0.919$ & $<0.001$ \\
\hline 9.Anhedonia & $0.88 \mathrm{I}$ & $0.796-0.943$ & $<0.001$ \\
\hline I0.Flattened affect & 0.875 & $0.773-0.95$ I & $<0.001$ \\
\hline II.Worthlessness & 0.854 & $0.729-0.944$ & $<0.001$ \\
\hline 12.Helplessness & 0.825 & $0.680-0.933$ & $<0.001$ \\
\hline I3.Suicidal ideation & 0.705 & $0.452-0.898$ & $<0.001$ \\
\hline I4.Guilt & 0.667 & $0.44 I-0.835$ & $<0.001$ \\
\hline I5.Psychotic symptoms & 0.873 & $0.727-0.950$ & $<0.001$ \\
\hline 16.Irritability & 0.715 & $0.461-0.876$ & $<0.001$ \\
\hline I7.Lability & 0.632 & $0.289-0.895$ & $<0.001$ \\
\hline 18.Increased motor drive & 0.800 & $0.527-0.961$ & $<0.001$ \\
\hline 19.Increased speech & 0.840 & $0.673-0.943$ & $<0.001$ \\
\hline 20.Agitation & 0.689 & $0.360-0.931$ & $<0.001$ \\
\hline BDRS & 0.930 & $0.880-0.967$ & $<0.001$ \\
\hline BDRS-D & 0.955 & $0.921-0.979$ & $<0.001$ \\
\hline BDRS-M & 0.791 & $0.619-0.915$ & $<0.001$ \\
\hline
\end{tabular}

Abbreviations: BDRS, Bipolar Depression Rating Scale; BDRS-D, the depressive subscale of the BDRS (itemsI-15); BDRS-M, the mixed subscale of Bipolar Depression Rating Scale.

We investigated the concurrent validity of the BDRS-C by computing Pearson correlation coefficients with other validated scales. In line with the previous findings, our data demonstrated that both the total BDRS-C scores and the depressive subscale scores had a strong positive correlation with the HAMD-17 and the MADRS scores. These results indicate that the scale performs equally well as the HAMD-17 and MADRS in accurately assessing depressive symptomatology in patients suffering from a depressive episode of bipolar disorder. In addition to evaluating depression, the BDRS-C assesses mixed symptoms of depressive episode of bipolar disorder, such as increased motor drive and speech. A moderate correlation was found when the mixed subscale was compared with the YMRS, as expected. This finding is very important since many patients suffering from a depressive episode of bipolar disorder have concurrent manic symptoms that must be evaluated, and the BDRS was designed to capture these clinical features.

Factor analysis confirmed that the three-factor structure of the BDRS-C is consistent with the construction of the original and other international validations, but the labels of the three factors and several items were different. In the original scale, the factors were loaded onto psychological depression (eg, social impairment, anxiety, anhedonia, worthlessness, helplessness), somatic depression (eg, depression, sleep and appetite disturbance, reduced motivation and concentration), and mixed factors (eg, psychotic symptoms, lability, and increased motor drive and speech). ${ }^{21}$ However, in our study, depression, sleep disturbance, appetite disturbance, activity/energy reduction, reduced motivation, anhedonia, flattened affect, worthlessness, helplessness, and suicidal ideation were classified as primary depressive factors. Most of these items are symptomatic diagnostic criteria of major depressive episodes from the DSM-5 and the International Classification of Diseases (ICD-11). Social impairment, reduced concentration, anxiety, guilt, and psychotic symptoms were grouped under the secondary depressive factor because they did not constitute the core symptoms of depression. The differences in depressive factors between our study and the original study ${ }^{21}$ may be associated with the clinical characteristics of the patients in two studies and cultural differences. It is well documented that depression symptom patterns are influenced by ethnocultural backgrounds. ${ }^{35}$ For Chinese patients, they tend to express more somatic symptoms. ${ }^{36}$ Consistently, the factor analysis in our study has shown that both the items including the core symptoms and somatic symptoms of depression have loaded onto the same factor (primary depressive factor). The items in the mixed factor included irritability, lability, motor drive and increased speech, and agitation and were the same as the Spanish validation ${ }^{30}$ and the Korean version; $;^{31,32}$ 
however, this was not the case in the original study, which included psychotic symptoms but did not include irritability. $^{21}$

In this study, test-retest reliability was used to evaluate score stability within a five-day interval. The testretest reliability of the total BDRS-C and the depressive subscale was excellent, and the mixed subscale was acceptable. The same analysis showed that the test-retest reliability of each item was acceptable except for three items (guilt, lability and agitation) with relatively low reliability.

This study presents limitations that need to be taken into account. First, the convenience sampling method was adopted, and the participants were all from one hospital. Therefore, the BDRS-C should be further verified in multiple regions. Second, we did not conduct structured diagnostic interviews to determine the diagnosis. Finally, all patients were on psychotropic medication at the time of the interviews, which may have impacted the item ratings.

In summary, our findings provide sufficient evidence of the validity of the BDRS-C. Consistent with the original scale, the BDRS-C presents satisfactory psychometric properties with good internal validity, interrater reliability and strong correlations with other commonly used depression rating scales. Therefore, it can be used in clinical practice and medical research, including evaluation of the clinical efficacy of new drugs, as a valid measurement instrument and a specific tool to screen and quantify the severity of depressive episode of bipolar disorder.

\section{Acknowledgments}

We thank all subjects who participated in this study. We thank all researchers and scientific advisors for their contribution to the design of this study. We thank Professor Yong-Sheng Tong for his guidance on statistics.

\section{Funding}

This research was supported by the Capital Health Research and Development of Special (2018-3-2132), National Natural Science Foundation of China (No. 81771468, 81601184).

\section{Disclosure}

The authors report no conflicts of interest in this work.

\section{References}

1. Ferrari AJ, Stockings E, Khoo JP, et al. The prevalence and burden of bipolar disorder: findings from the Global Burden of Disease Study 2013. Bipolar Disord. 2016;18(5):440-450. doi:10.1111/bdi.12423

2. Clemente AS, Diniz BS, Nicolato R, et al. Bipolar disorder prevalence: a systematic review and meta-analysis of the literature. Braz J Psychiatry. 2015;37(2):155-161. doi:10.1590/1516-4446-2012-1693

3. Huang $\mathrm{Y}$, Wang $\mathrm{Y}$, Wang $\mathrm{H}$, et al. Prevalence of mental disorders in China: a cross-sectional epidemiological study. Lancet Psychiatry. 2019;6(3):211. doi:10.1016/S2215-0366(18)30511-X

4. Zhang YS, Rao WW, Zeng LN, et al. Prevalence and correlates of bipolar disorder in the adult population of Hebei province. China J Affect Disord. 2020;263:129-133. doi:10.1016/j.jad.2019.11.104

5. Kupka RW, Altshuler LL, Nolen WA, et al. Three times more days depressed than manic or hypomanic in both bipolar I and bipolar II disorder. Bipolar Disord. 2007;9(5):531-535. doi:10.1111/j.13995618.2007.00467.x

6. Judd LL, Akiskal HS. Depressive episodes and symptoms dominate the longitudinal course of bipolar disorder. Curr Psychiatry Rep. 2003;5(6):417-418. doi:10.1007/s11920-003-0077-2

7. Judd LL, Akiskal HS, Schettler PJ, et al. The long-term natural history of the weekly symptomatic status of bipolar I disorder. Arch Gen Psychiatry. 2002;59(6):530-537. doi:10.1001/archpsyc.59.6.530

8. Baldessarini RJ, Tondo L, Pinna M, Nunez N, Vazquez GH. Suicidal risk factors in major affective disorders. $\mathrm{Br} J$ Psychiatry. 2019;1-6.

9. Miller JN, Black DW. Bipolar disorder and suicide: a Review. Curr Psychiatry Rep. 2020;22(2):6. doi:10.1007/s11920-020-1130-0

10. Au JS, Martinez de Andino A, Mekawi Y, Silverstein MW, Lamis DA. Latent class analysis of bipolar disorder symptoms and suicidal ideation and behaviors. Bipolar Disord. 2020. doi:10.1111/ bdi. 12967

11. Patella AM, Jansen K, Cardoso TA, Souza LDM, Silva RAD, Coelho F. Clinical features of differential diagnosis between unipolar and bipolar depression in a drug-free sample of young adults. $J$ Affect Disord. 2019;243:103-107. doi:10.1016/j.jad.2018.09.007

12. Leonpacher AK, Liebers D, Pirooznia M, et al. Distinguishing bipolar from unipolar depression: the importance of clinical symptoms and illness features. Psychol Med. 2015;45(11):2437-2446. doi:10.1017/S0033291715000446

13. Vazquez GH, Lolich M, Cabrera C, et al. Mixed symptoms in major depressive and bipolar disorders: a systematic review. J Affect Disord. 2018;225:756-760. doi:10.1016/j.jad.2017.09.006

14. Bowden CL. A different depression: clinical distinctions between bipolar and unipolar depression. J Affect Disord. 2005;84(2-3):117-125. doi:10.1016/S0165-0327(03)00194-0

15. Benazzi F. Depressive mixed state: a feature of the natural course of bipolar II (and major depressive) disorder? Psychopathology. 2004;37 (5):207-212. doi:10.1159/000080715

16. Diler RS, Goldstein TR, Hafeman D, et al. Distinguishing bipolar depression from unipolar depression in youth: preliminary findings. $J$ Child Adolesc Psychopharmacol. 2017;27(4):310-319. doi:10.1089/cap.2016.0154

17. Nunez NA, Comai S, Dumitrescu E, et al. Psychopathological and sociodemographic features in treatment-resistant unipolar depression versus bipolar depression: a comparative study. BMC Psychiatry. 2018;18(1):68. doi:10.1186/s12888-018-1641-y

18. American Psychiatric Association. Diagnostic and Statistical Manual of Mental Disorders(5th ed.). Washington DC: APA publishing; 2013.

19. Montgomery SA, Asberg M. A new depression scale designed to be sensitive to change. $B r \quad J$ Psychiatry. 1979;134(4):382-389. doi:10.1192/bjp.134.4.382

20. Hamilton M. A rating scale for depression. J Neurol Neurosurg Psychiatry. 1960;23(1):56-62. doi:10.1136/jnnp.23.1.56 
21. Berk M, Malhi GS, Cahill C, et al. The Bipolar Depression Rating Scale (BDRS): its development, validation and utility. Bipolar Disord. 2007;9(6):571-579. doi:10.1111/j.1399-5618.2007.00536.x

22. Hantouche EG, Akiskal HS. Bipolar II vs. unipolar depression: psychopathologic differentiation by dimensional measures. $J$ Affect Disord. 2005;84(2-3):127-132. doi:10.1016/j.jad.2004.01.017

23. Samalin L, Boyer L, Murru A, et al. Residual depressive symptoms, sleep disturbance and perceived cognitive impairment as determinants of functioning in patients with bipolar disorder. $J$ Affect Disord. 2017;210:280-286. doi:10.1016/j.jad.2016.12.054

24. Khosravani V, Mohammadzadeh A, Sharifi Bastan F, Amirinezhad A, Amini M. Early maladaptive schemas and suicidal risk in inpatients with bipolar disorder. Psychiatry Res. 2019;271:351-359. doi:10.1016/j.psychres.2018.11.067

25. Berk M, Dodd S, Dean OM, Kohlmann K, Berk L, Malhi GS. The validity and internal structure of the Bipolar Depression Rating Scale: data from a clinical trial of $\mathrm{N}$-acetylcysteine as adjunctive therapy in bipolar disorder. Acta Neuropsychiatr. 2010;22(5):237-242. doi:10.1111/j.1601-5215.2010.00472.x

26. Pandya SP. Meditation for treating adults with bipolar disorder II: a multi-city study. Clin Psychol Psychother. 2019;26(2):252-261. doi:10.1002/cpp.2347

27. Quide Y, Bortolasci CC, Spolding B, et al. Systemic inflammation and grey matter volume in schizophrenia and bipolar disorder: moderation by childhood trauma severity. Prog Neuropsychopharmacol Biol Psychiatry. 2020;110013.

28. Shabani A, Akbari M, Dadashi M. Reliability and validity of the Bipolar Depression Rating Scale on an Iranian sample. Arch Iran Med. 2010;13(3):217-222

29. Batmaz S, Ozdel K, Kocbiyik S, Karadag H. The validity and reliability of the Turkish version of the bipolar depression rating scale. Compr Psychiatry. 2014;55(6):1448-1454. doi:10.1016/j. comppsych.2014.04.020
30. Sarro S, Madre M, Fernandez-Corcuera P, et al. Transcultural adaption and validation of the Spanish version of the Bipolar Depression Rating Scale (BDRS-S). J Affect Disord. 2015;172:110-115. doi:10.1016/j.jad.2014.10.009

31. Jung YE, Kim MD, Bahk WM, et al. Clinical assessment of bipolar depression: validity, factor structure and psychometric properties of the Korean version of the Bipolar Depression Rating Scale (BDRS). BMC Psychiatry. 2016;16(1):239. doi:10.1186/s12888-016-0958-7

32. Lee DY, Won EK, Choi J-W, et al. Feasibility of the Korean version of the Bipolar Depression Rating Scale in adolescents with early-onset bipolar disorder. Psychiatry Investig. 2017;14 (5):585-594. doi:10.4306/pi.2017.14.5.585

33. Bruschi A, Mazza M, Camardese G, et al. Psychopathological features of bipolar depression: Italian validation of the Bipolar Depression Rating Scale (I-BDRS). Front Psychol. 2018;9:1047. doi:10.3389/fpsyg.2018.01047

34. Young RC, Biggs JT, Ziegler VE, Meyer DA. A rating scale for mania: reliability, validity and sensitivity. Br J Psychiatry. 1978;133 (5):429-435. doi:10.1192/bjp.133.5.429

35. Ryder AG, Yang J, Zhu X, et al. The cultural shaping of depression: somatic symptoms in China, psychological symptoms in North America? J Abnorm Psychol. 2008;117(2):300-313. doi:10.1037/ 0021-843X.117.2.300

36. Zhu L. Depression symptom patterns and social correlates among Chinese Americans. Brain Sci. 2018;8(1):16. doi:10.3390/ brainsci8010016
Neuropsychiatric Disease and Treatment

\section{Publish your work in this journal}

Neuropsychiatric Disease and Treatment is an international, peerreviewed journal of clinical therapeutics and pharmacology focusing on concise rapid reporting of clinical or pre-clinical studies on a range of neuropsychiatric and neurological disorders. This journal is indexed on PubMed Central, the 'PsycINFO' database and CAS, and
Dovepress

is the official journal of The International Neuropsychiatric Association (INA). The manuscript management system is completely online and includes a very quick and fair peer-review system, which is all easy to use. Visit http://www.dovepress.com/testimonials.php to read real quotes from published authors. 